\title{
3D Design Remodeling with Idealization Approach
}

\author{
Aykut Zongur \\ Project Directorate Office, Computer Engineering, State Ministry, Turkey; \\ z_aykut@yahoo.com
}

\begin{abstract}
Objective: Our customization of design works is embroidered by a bundle of pre-post structural processing's over unprocessed finite fields in redesigning the modelings for performance with proprietary quantitative approaches. Our boundary-adaptive refinement operations pass through a special-in-design boundary adaptations for computerized design working. Methods: The context-varying remodeling continuum is customized by sewing up of the compound betwixt the interseams proceeding by the combinations of that in order to make a retouching statement with an unprecedent outfitting capability, respectively. Stopping-criterion refinements with self-adaptable boundary adaptations have been geared up to a certain extent of degree for rendering at finer timing axes. Augmented realizations of redesigned models have been manufactured in high-realism that is unmatched by any other on-shelf softwares. Our hand-made remodeling customizations are highly refining the visualizations of shape editings in fineness terms and in context of content-richness by means of both time and fidelity factor either. Findings: While remaking the models, nothing is forfeited neither from time nor from the reality at both hands of the dimensions. Our procedural designings are for serially taking the remade models to the upper bounds of the reality dimension. Refinements are calibrated in timely manner by no means of delays in the course of building procedures. Our model recreations are remodeling the starters without the exchange of virtual reality to serial-remakings. The restorative realizations are made within the conservation of realism by getting out of authenticity. Surpassing the trade-offs make the proceessed models realistically drape like waves while being on execution either. Application: Conclusive outcomes are at ideality-added state by being not only quicker but also far realistic findings from here on.Our creative model designing for performance is at the highest-standards with our chain of proposed techniques which are qualified in a way that have never been offered by an almost any design and simulation systems ever.
\end{abstract}

Keywords: Computerized Modeling, Morphology Edition, Restorative Model Redesigning, 3D Shape Remodeling, Virtual Augmented Reality

\section{Introduction}

Nonlinear rational B-splines (Nurbs) even themselves do predictably pose challenges which were left behind the scenes under the provisions of mass-spring schemes ${ }^{1}$. Finite fields are capacitated with multiple splinegons even when making them drape like seamlessly as nonartifacts. Our remodeling processes are destined to configure out the outfit models as much authentic as possible. Hybrid concepts of geometrical plus physical configurations will have outlooks as if likean illusion². The configurated granularity turned out to be fine-granular inasmuch as it can be. Our layered structure must be draping in a way that the distinguishing outlines of the models became hard to be differentiated. 
Past works are much related with taking on the surroundings ${ }^{3}$. Several many former methods were proffered for general-in-refinements but none of them was able to exceed our current rigging skinning reconfigurations. These are possibly the hotbeds of the near future ${ }^{4}$. Complex nurbs blending is also an extra-factor for the restorative treatment of layered-models: Reformations get to there whenever the deformations gets out of control in response to the reforms that were to be made on the geometrical topology ${ }^{5}$.

Active mesh editions were essential for the lock points of the meshes that are laid down on the layouts ${ }^{6}$. Mesh reformations are brought in the wake of an all-in-one sort of approaches and hands the element-rich surfaces over there the areas right here. The destination was speed and quickness in modeling and simulations. Eye catching outcomes of the interested areas have been at the view of the show cases since from the first initial start. Our tailoredfittings are executed on the road to the final check outs. Virtual models would be emulated by being fitted onto the surfaces ${ }^{\underline{z}}$. States of art exhibitions were derived from the object model designs over all the nurbs of free body objects ${ }^{\underline{8}}$.

Right content meshes are rebuilt on the surfaces of the models right here. It is just as the way the full-rank coverage of high-complexity structured modelings are made $\stackrel{9}{ }$. Renewals of contents are lied over the over ground flooring. Inner faces of nurbs for the outer surfaces are yet to be redone in the theme of model renewals. Status ofthe meshes is reconditioned with the boundary conditions over the construction sites being tested on. Try-on tests are carried over direct-3D modelings for the trained data sequences ${ }^{10}$. Much has been done for exploring the faces of surfaces, despite being not limited to model characteristics only. When it comes to calibrating the resolution arrangements along side the wave nurbs for the training operations were not lonely the only issues neither ${ }^{11}$. Vivacious outlooking is obtained from away whether exemplification arises or not while being draped over free body models. Having content-richer designs for the highly-realistic models have become far beyond the services offered by today's most commercial design softwares ${ }^{12}$. Our specially designed systematization is for the next-level of meshing with buoyant configurations. Outlooks of scenery have much more buoyancy rather than everywhere else in here ${ }^{13}$.

\section{Regulation Gauges}

Knitting the faces of models could possibly be effectuated with our 3D spatial continuitization of piecewise polynomial spaces for 2/3-dimensionality states. Assembly of subspaces is exactly approximated by being splined over the nonlinear approximations of supspaces such like as in the space ranges. Continuous structures are rebuilt in a way that takes our system work to higher dimensions of closed-form meshes. Installment of the lay-up splinegons on polygonal model areas would be a handcraft to follow especially when the floor complexities are at the highest levels recently.

Boundary conditioning encloses the surroundings from the perimeter. Models are to become attainable by directly reforming the global meshes in local scale. Transformation of local meshes into global can even become possible by our methods lastly. Locales are reformed with point set of clusters. Interelemental faces are situated on the corners of edges. Corner nodes cannot move over any other polygon edges. Nodes are not always equal to the vertices of elements. Surrounding wingspan of topology is ranging from inter-(ior) to exter-(ior) borders, respectively. Gauge for elements is calibrated on the local extent (the greatest edge component).

The gauges of meshes are globally calibrated at an extent of $a=\max T(a)$ in wide extents. Global area mesh finenesses exit from origin of block structures. Adaptive refinements are gauged by our following regularities of triangularity right down:

$$
b_{T}=\frac{a_{T}}{r_{T}}
$$

subject to;

$$
b_{0} \leq b_{T} \text {, on } \forall \mathrm{T} \in \overline{\mathrm{T}}
$$


Where $r_{T}$ and $a_{T}$ are the diameter of the circle of local grid cell, respectively. $b_{T}$ is the bullion parameter in where elements are scribed to theriggings.

Condition-zero of boundaries prevents polygon nodes from excessing the boundary values by triangularinequality violation avoidance. Piecewise function spaces have been grounded on the starting line-up of points that are to be netted in demarcation zone meshing. Boundary conditions are initialized at the upturn of initial values on equality basis. The elliptical differential equations are set out for the regular-shaped modeling. Boundary values might fall inside or outside the intended areas of interest in the ranks of initialization.

Procedural polygonization is spatially meshing the all tri-states of models by our triangle mesh size $s_{T}$ for valid number of elements in variations. Conditioners in our system are well-conditioning the borders on both ends of the spatial and time axes. Having been preconditioned the place-variant spatial conditions, inequalities were evolved into the followings right here:

$$
1 / \lambda<\frac{a_{T}}{a_{T^{b}}}<\lambda, \text { on } \forall \mathrm{T}^{\prime}, \forall \lambda>0
$$

Sort of point models have been selected with $n_{p}$ nodes of the adjacencies for the finite fields which are held at matrix $A$ consecutively.

$N$ is a size of $3 \times n_{n}$ node and $A$ is a $4 \times n_{t}$ adjacency matrices. (Counter)clock-wise partial orderings have not got much difference in model formations. These ordering options would probably be distributive but not associative nor commutative neither. Notations in general are also valid for the finite spaces of functions. Polygonal fields are restored at tetrahedral holdings in particular. Iso-partitioning involves four rows to four columns of $N$ in matrices $A$.

\section{Calibration Adjustments}

Proposed chain methods have enabled our netting scheme to yield richer areas in semi automatical way. Skins are armoured by an overlay system work in a complete fit- ting. Analytical planes are fully refulled in a moment. Automated workflows is customized for fully filling the contents of meshes. Special status of meshes could get far richer by this way. The shapes of our surface models were still to be refactorized until it has yet evolved into that sort of dimensionality. Multi-dimensionality modeling are to be conditioned within mixture of boundaries on where the 3-decker buildings pass over there.

Majority of groundworks have been initally set off from the initial start at the Figure 1. Level of regularity for the calibration functions had been taken to intended levels on ground surfaces. Initial point values are overlaid with support hat functions of any point mappings on ready. Our multi-resolution plans invoke the nurbs that would be interconnected at any time soon. Finding the equilateral polygons equilaterally is rare and unusual in practice. Our model design passes over each corner of lines at the cross sections of the edges. However, these were not optimally the greatest of all in usual. Elimination of irregular items were underway around the boundaries of the model areas after regulating them by our series of

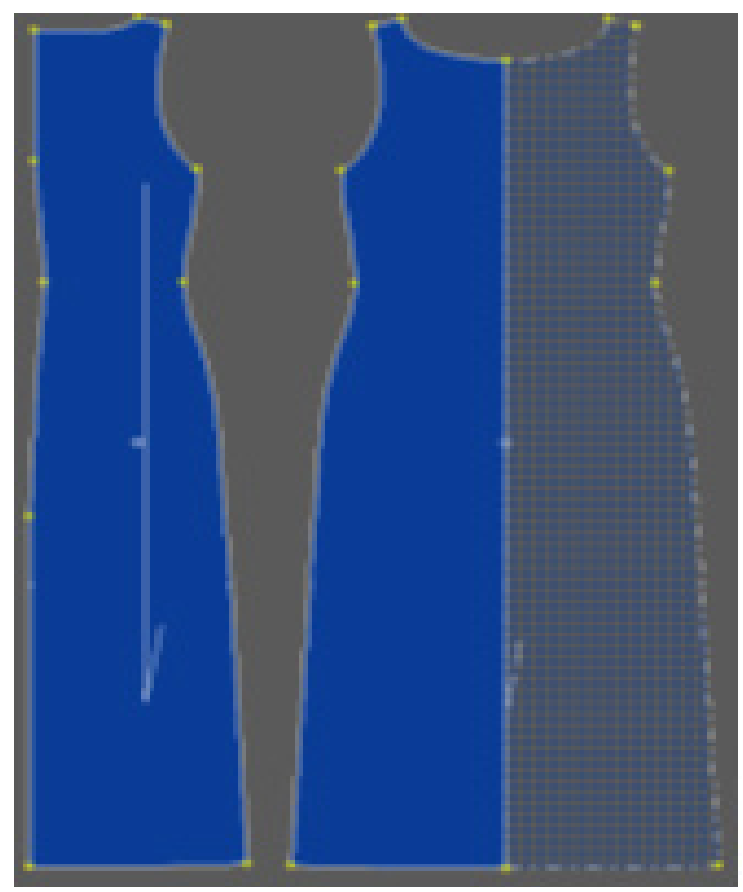

Figure 1. Initial state formations. 
geometrical processings. Regulations for model designing has built-in well-established isometries.

Design essentials are found for architectural modeling. Either of the rotated $\mathrm{R}(\mathrm{ight}) / \mathrm{L}$ (eft) parts are the twinning components of any reshaped compound. Involvement of direct design would mount the parts in time of sewing over any work benches. Structured tiling in tetrahedrons have neither been quadrangulated yet.

Our assembly functions make the mountages of any given cluster of points uniform. Adaptive distance functions fetch the maximum of edgesat all. Our manufacturing contains a node matrix $N$, an adjacency matrix $A$ and an edge matrix $E$ involving the all nodes of edges in addition to the number of polygon facesat there. Secrecy of Ematrix is implicitly hidden in the closed-form models.

\subsection{Building-blocks Arrangement}

Our multi-purpose methodology assures the isometry of the polyhedral blocks. Newer topology is actively set on the newly-formed meshes having its own initialization procedure in itself. Our working utilizes from the different sparsities of density conditions. There are also mid-dense settings being found in wider extents. Topological configurations have been restructuring by our modified tetrahedral tilings over progressive interpasses. Boundary delimitations also are cut out from there either. Reconfigured topologies are found in continuous time-series for constructive solid geometries.

All argument types are controlled by our functional controllers in time varying modes. Numbered one priority related to here is the calibration of the interested areas. None of the convex polygons prespecified check points of themselves is greater or even equal than the original number of point nodes. The growth and the stability factors of meshes are there for the calibration adjustments with element settlements. Interchanging values of multi-dimensional grids are ranged in between the preceeding upper-lower bounds. On-site calibré controlsof the surroundings is worked on enclosurement working pitch.
All sorts of cross-checks over the frontlines does also in close the control points to there. Each exception like getting out of bounds of there is seperately handled by hand.

Algorithm_of the polygonization process of splinegon surfaces.

- $\quad$ construct (Equation 5);

- $\quad$ polygonize (nodes);

- for Equatioin 6 do

- $\quad$ for edges $!=$ spline ${ }^{+}$do

- $\quad$ encirculate $\left(a_{T}, r_{T}\right)$;

- $\quad$ end for

- $\quad$ end for

- $\quad$ destruct();

Raw surfaces are handed over our phasing processes. Set of vertices are to be replaced by the node points. The topology in general is surrounded by exterior zones. Polygonization of the edge points will be staged up in turn. Newest established conditioning is worked out to make bound-checks of the upper-lower boundary limits. Node vertices are assembled to construct the finite spaces on the ground of block centers inside areas. Final set on range has been made movable while our construction on the way is underway. Our multi-gridding would culminate in multi-dimensional grids while cultivating the finite fields.

Here is the annotation of ${ }^{+}$annotates the transition phases of the splinings. Two-phase passageway takes stage intransitory passes from one to other. B-splines are brought about when the interpolants have been interpolating 14 . The B-type of splines crosses over each cutting edge of the polygon lines in transit. One on one inversion of transitive splining reverses the course of phasing in an irreversible way. These nonlinear transitions are splining the each interpolant of the lines in backward directions. 


\subsection{Angular Calibrations}

Extent of fineness of meshes was directly forwarded to the manual manipulation. By upsampling the preceedings, the mould works is gauged with our special-in-calibrations. Structuring blocks for grid elements such as $\mathrm{N}$ nodes, $E$ edges, $\mathrm{T}$ triangles are the ones that have been made use of. Topological calibrations for trio-restructuring are calibrated by our special-defined quantifiers:

$$
\text { c. } v=\frac{a^{2} \sqrt{3}}{3 h_{a}^{2}}
$$

Where $h_{\mathrm{a}}$ are the projected heights of the equal sides of the $a$ polygons. Calibrator functions are reforming the model-fineness in the formatted intervals of models. Model values are the factors for the fineness of calibrated grids on view.

\section{Self-adaptations}

Interconnected meshes is revealed to be elliptically-posed differential systems that are to be functioning for the exact solution of these interconnections:

$$
-\nabla \cdot(a \nabla u)+b \nabla u+c u=f \text { on }[\Omega]
$$

Alternative descent of rising gradient values arises out of linings from each center of polygons. $a, b$ and $c$ are the characteristic properties of the equational finite dimensional spaces. $\mathrm{u}$ is the probability of vector valued solutions to the problems of here. $\Omega$ are the borders that have been worked on the problem domain but also for the solution candidates followed by sequence of lay-up splines in progressive ongoing. Polygonal models are adaptively refined by our finite differential schemes with some particular selections of elements. Differential systems have been both iteratively and recursively resolved for maximum accesible resolutions.

Refinement resolution proposals will further on by our polygonal reworkings until any newer parcel can not be found to locate in anywhere else. Our specialization is peculiarly functioning for ad-hoc meshing. Topological errors of the high-resolution meshes have transit passed to the adaptive regulations with the inclusion of the redefinition of selection functions. Redesigning of meshes for performance is to be equalized at a caliber of world-class service unlikeas before. Renewal of updated coefficients for the polygon models are effectuated in the final recreations just as in Figure 2.

Spatial continuatizations are ever-renewing in varying conditions. Up-to-date resolutions are found out along all across the wide area fields. Calibrator adaptations have been getting into track. Error estimations are there with the multi-criterion equalizers selections in currency. The inequalities are poised just as indicated on the domainspecific axes down here:

\section{ester $=$ residual $>$ limit golden_ratio;}

$$
\text { golden_ratio }=\max \left[\max (\vec{f}) * h^{2}, \max (\vec{a}) * \max (\vec{u}) * h^{2}, \max (\vec{c}) * \max (\vec{u})\right] ;
$$

Where $h$ is the minimum sides of all polygons in largest extent and $g r$ manipulates the whole model boundaries. Post makings would last till the element amounts have arrived to highest possible volume capacity. Processes would get out of execution whenever the number of polygons reach to the upper bound limits of differentials. Nonlinear equations are to be linearizing the transformation coefficients in the genera lsolutions.

The finite space arrangements are much dependent to the spatial axes for that. Transformations are bounded by the solution spaces that have been approximating to. Explicit parametrizations are consisting of timedependency factors. Refinements are not limited to the variations of mesh events. In-house rectifications of the models were bounded to the restricted free boundary constraints. Covering line-ups were to be versioned through our boundary adaptations on there. Borders are quickly made adapted to nonlinear partial differential equations 


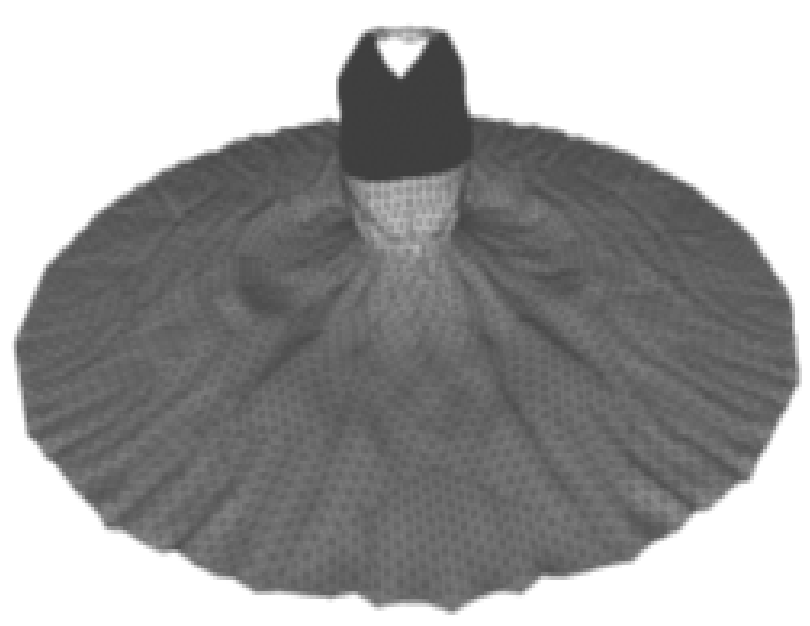

(a)

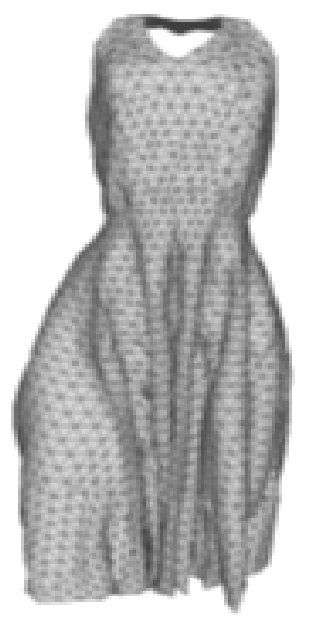

(b)

Figure 2. Tailored models are uniformly post-processed in time-variances. (a) Pre-processings in space revolve over there. (b) Hulling after post-processing actions.

at the time of self-adaptive refinements for nonlinearity. Adaptivity of tolerance levels is necessarily refined with starting initial value conditions in nonlinear editions. Residuals here are passing by values in time varying form of adaptations over the passage of iterations.

Geometrical calibration works are cut into manual adjustments for our 3D remodelings. Design particulars are optimally automated in our specific ways.

\section{Automatic Refinements}

Node points are numbered to be driven through procedural refinements. Numbered nodes which have been under alignment are indexed with some particular locations of the surfaces. Cluster of vertices are called by their index numbers of polygons. These clusters are controlled by the point indices on all across the fields. The retouches gets and returns the elegant topology in turn. Premier working passes in sequence of refineries while out doing the former states of arts. Here our refinery works have extended out the building structures to time invariant higher dimensions of all. Reform series extensions were done with our extensive way of finer granularities. Nonlinear splines are optionally laid on the faces of surface areas. Dyanmic duos are uniquely cascaded in external-internal styles of reformations.

Our groundworks for the multigrid isometry pass beyond the baselines of model works in varied geometries. Rectifications of surface shapes are here on the ground of fineness of elements. Solid models are partitioned into variety of model segments like as in the combined states of modelings. Polygonal model faces are yet to be refined by the maximum of all that would have to be segmented into sub units. Both quickness and speedness were to be configured on the base of convergent splines instead of divergent polynomials. Delimitated divergencies would be completely filled out in fullness. There has been the liability of the regulation of unpolygonized sectors of which are being worked on. Elegancy inside the exterior boundaries of topology will be so advanced till the last hanged up nodes are to be restored. Demarcated polygonizations are caught up at an intended regularity of fineness in the related area out of bounds as like in the Figure 3.

Seatingof the equi-sided polygons as isohedrons was not a mandatory nor a compulsory for anywhere else excluding here. However, the necessary and sufficient conditions for the equality of polygons were always on 


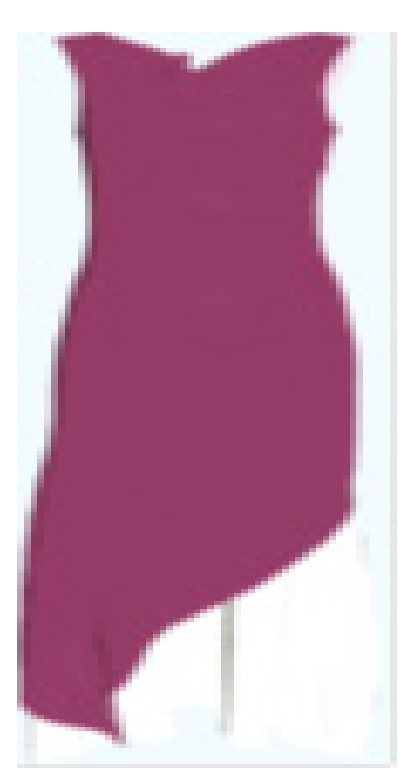

(a)

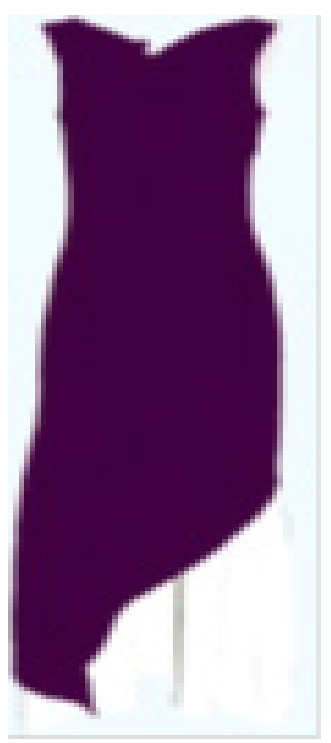

(b)

Figure 3. (Un)processed fabric surfaces collocates with:a. Initial state of the hull right before passing to hulling processings. b. Hull-liners have undergone a passage of the post-processing.

our agenda to have them in proper seating order. Seating of the right faces on the right edges have always been a priority for the regular seating arrangements. Wide range of relocations are to offer exact regulations for the belonging trailer elements of there. Interelemental equality checks are for isohedral meshing. The resolutions have been enhanced in the post-processings of highlyoptimized refineries for boundary conditions. Resolution highness is proportional to regularness. Maximum number of iterations is in direct proportion to multiresolutions in any way. A priori arguements of optimality are returning in maximum iteration number. Execution is proceedingly forwarded to an optimal value of predefined iterations limit which is bounded to the boundary edges of there.

$$
W=\underset{W}{\operatorname{argmax}}\left(\sum_{i}\left\|W \cdot \vec{\alpha}^{i}+\vec{\beta}^{i}\right\|^{2}-\lambda \cdot\|W\|^{2}\right)
$$

Where an adaptation-varied model refinement takes places. $W$ is the power matrix of an aweighted linear system of equations. $\alpha$ exists for the organization of shape models. Existence reason of $\beta$ the-structuring-elements is for enriching the models in general. $\lambda$ is the Lagrangeconstraint value to there.

$$
W \cdot \vec{\alpha} \leq \vec{\beta}^{n}
$$

Where $N$ is an armour-clad numbering. Shape models are refined over our progressive enrichment plans. Parcelizations way pass from our built-in constructors for the upsampling additions of any unit elements. Weight-factored nonlinear systems are passed to the refactorization on our current base space of finite structured-elements.

Faces of models are relocated on the element edges they are relying to. Each element locations are on the edge-leaning lines. All elements are collocated to contiguous edges of each particular location of meshes. Renewals 


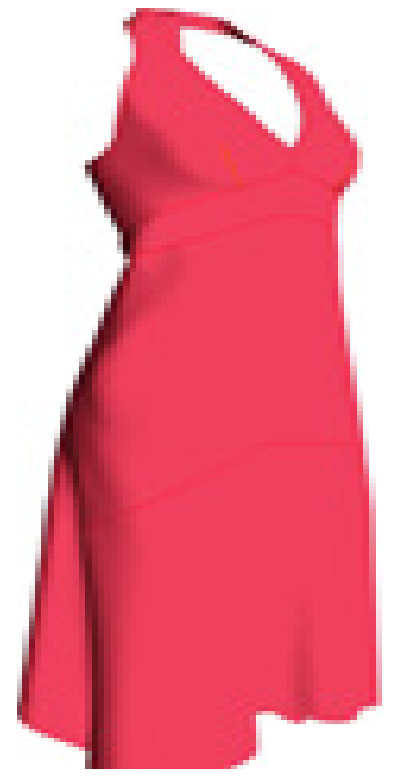

(a)

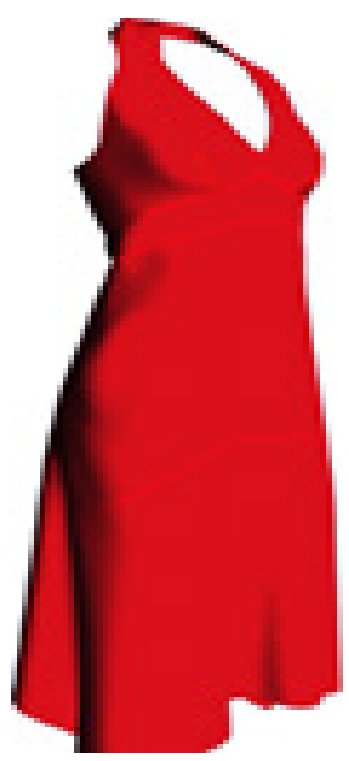

(b)

Figure 4. Unprocessed vs. processed overground layouts.

have much to do with adjaciencies which are passing from the inscription centers of there. Both a prior and a posteriori conditions are controlled over the boundaries of the zone area since the process is streaking on our golden ratio base. Registered fineness of overall model layouts have been much increased in the continuation of processed modelings. Our process of overlay meshes will not stop unless much amelioration is lived in the fineness of the models of surfaces just as examplified in the Figure 4.

Highest delicate modelings became attainable by our proposed chain of techniques. Our resolution offer was on the wayback to the check-outs for the boundary crossings. There are not many hanging points being left there either on global and local extents in the assembly of model segments. Robust pre-processings were effectuated in the early phases of these. Self-adaptations have cut in when not much elegancy-advancements have been progressed there. In the flow of the process, these ongoing processes could not infinitely run to the infinity which is being passed over.
Axial annexations were for manually selecting the best fitting on the axes of free 3D models. Splining of interpolants with b-type splines is gradated by all upperlower boundary crossovers on the initial value conditions. Concepts of our remodelings are taking the starting linings to the extension models by further than the passed continuum.

\section{Experimental Results}

Realism of our reinforced modelings is in golden ratio with the new ancillary parcelization plans. Newly reformed meshes will never be the same saving the ones in general contours. Range areas have been getting armoured with a highly affluent armoring. Overlay parcelization is retouched with an overall rebuilding on range areas.

Our ground working fills the elemental-lacknesses of open fields for the high-resolution configurations in modelings. Area zoning is generally configured in cure of the best possible option that could be yielding an internal proliferation in overall. 
The resultant outcomes had forward-looking manufacturing by an internal restructuring that reshapes the ultimate models. Finest state of arts is modulated in finalfashion for any random walks on the runways. Stylistic designs have been enabled for wayout outlooks by the assistance of our proprietary techniques. Fabricated recreations got armoured for realistic outer appearances such as having an original ground area. If fine art concepts are to be realized, our artwork will be projected out as the maximum possible fineness that can be achieved in ground covering. Flooring areas would have a realistic outlook from facade. This realistically draping conforms with designing and modeling practices of originality stencils. The closer the business gets to reality, the authentic the results will be at hand. All of these will be in such a way that would have added additive-reality to the essentials of them. Original states of our template configurations have been that much improved to mould the surrounder textures. Realism would have been unmatched if the iterations were far advanced with our further progressions rather than the latest models of the others which were once being tested in the early stages of the past plans.
Our resolution refinement offers are for taking the samples to higher resolutions without an extra support functions in line for peculiar (un)signed distances. Edge interconnections are so as to an self-made propellers on there over the interchange axes. Adaptive refinements for the reconstructive model regularization gets into the boundary-optimized rebuildings for there, Vertexformed training sets with test set-forming polygon edges are cross checked in manual. Our executive modelings for filling the reconfigurations were tested out with both the computational and realism factors regulations of boundary adaptations. Finite fields are capacitated in contents of element-wealth. Values of a priori and a posteriori estimates are proved to be error-proof over these estimations. Attributions of our methodology can add value to such recreations on the base of reference models that have been handing over from the formers to latter ones subsequently. Virtual models are built up in the frame of our past initial values subject to the boundary value conditions for an overall lay-down just as it is sampled in the Figure 5.

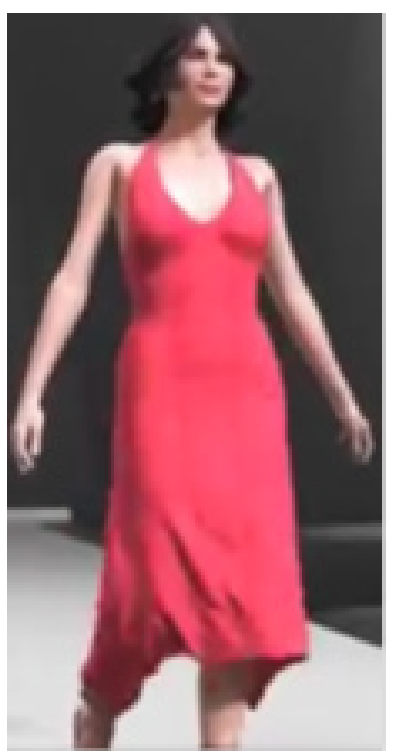

(a)

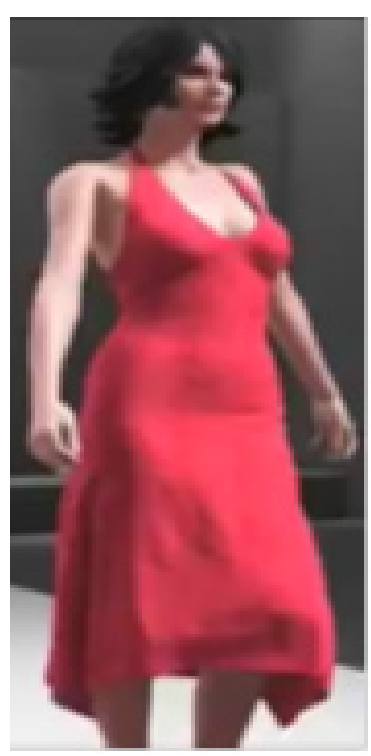

(b)

Figure 5. Modeling simulations were quickened while making use of interadaptations in manufacturing water-proofness. Frames from runways are accelerated by an optimal inter-refinements in the early times of modelings. Growth and the stability factors were adaptively brought to the equilibrium point. 
Meshes-in-general were renewed by the new high-resolution parcelings that lies over our modern parcelization system. The rational nurbs of the tri-axial planes are carried over the maximum allowable high-dimensionality. All these nurbs for here are in transit pass to high-dimensional rebuildings. Fully automated restorations with our mass-constructive refinements cross the high-dimensions along there.

Stringing points which are posted up in the model surfaces would turn out to be defunctory in the final state of redesigning. Here of our framing for the reworkings is specifically getting done in our multi-state parcelizations. There is not any more room left for the defunctory ones that have been hanging up by there.

One of our main intentionswas to dimensionalize the model shapes over the dimension axes at the start. All the dimensions were parcelized in manual self-servicing mode. Certain amount of batch data are post-processed as our element items of the meshes. Element collections have been collocated with the listed nodes at the boundary lines. Elemental finenesses are in relation with the restrictive spline collocations of the lowest minimum angle of boundary values. Our meshing works in operations are directly operating in finite element spaces.

Unsteady boundary conditions of the mesh zone induce to declines in the delicacy of the ground-zero. Surrounded surface models with mean curvature are relatively adapted to the exterior boundaries on the frontlines of outer region.

Model surfaces will be finer in the current form than in the start state of it. The final set on lines were crossed over the boundary edges. Our reformed models are made up of high numbers of elements. The construction areas have been specially reconditioned by our functional builders. Computational costs do majorly originate from the degradations from the elegancy of the organization areas. Reversal of points direction to other ends was a special one for multi-tasking operations. Adaptive refinements of the cluttered floors pass in wavy-way. Embroidery knitted-in parcels have become well-organized as for the full completeness of the surfaces. Designed states of modeling have been guaranteed with direct adaptations of the edges. Well-established states of arrangements have adaptively reconditioned the overall model formation elegances like as seen from the Figure 6 .

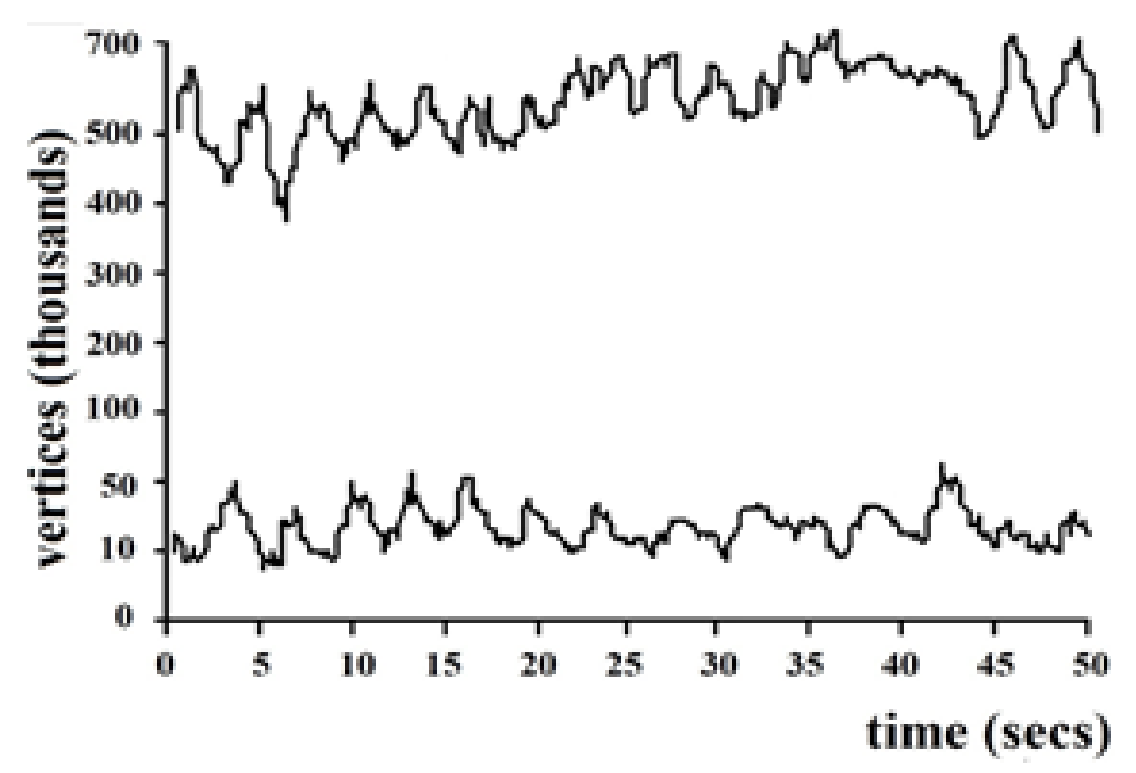

Figure 6. Vertice numbers for the pre-post processed states of our adaptive refinement designings are sketched up in chart. 
Table 1. Analysis of high resolutions are exemplified herewith our exhibition displays

\begin{tabular}{|c|c|c|c|c|}
\hline The unveil of the Model & \# Vertices & \#Triangles & Single (sec/frame) & $\begin{array}{c}\text { Animation (sec/ } \\
\text { frames) }\end{array}$ \\
\hline Initial State & 10693 & 17377 & 47 & 29 \\
\hline Processed State & 587794 & 1112128 & 38 & 22 \\
\hline
\end{tabular}

Computer workstations, by which the results were taken from, have got a video card owning an unshared 2-GB memory allocation on-port. Technical riggings are running over $2.00 \mathrm{GHz}$ dual-quad core processors supported by 8 GB-RAM as with our computational modelings for the displays of exhibitions.

Best of the visualizations are exhibited with exhibition displays and were parametrized either by an explicit scheme for nonparametric feature estimates. Our design modelings are cutting the edge of the times of post-processings. Post-up of high views on-site is of the direct 3D resolutions majorly. Our design work could have rarely set out newer settlement standards on the construction sites. Assembly of viewports on the work benches was set to standardize the net exhibition glidings for display. Showcases of the ground truths for the field works are of the latest states of over ground buildings which have been arrived to.

New designs of models are led to fashionable exhibitions by our content-rich modelings. Surfaces are dimensionalized over infinitely-size dimensions to qualify towards restrictive boundary conditions. Recreations have become rich-in-elements and still gets richer of elements in content. Models are assembled to meet the golden ratio of design fidelities. Rich content meshes are under service of augmented reality from this point on. Dimensionalizations are found for visually-realistic spatial optimizations of initial condition values that have been initialized with. Our system's weaponary is in building an infinitely-variable dimensional-specific customizations. Modelings have been serialized to an assembly line of model manufacturing that is posted upside our star gate display without much major forfeit from the realism. Visually good-looking models are created by our rebuilder designing in high octane stylejust likely in the Table 1.

\section{Conclusions}

In the highlights of this study, one full system workfor the $3 \mathrm{D}$ remodelings of virtual models is portrayed with our refinery-powered retouchings. Our optimized-customizations of fineness and time phonemena do culminate in an optimality-added work system as fine and soon as possible. Surface shapes were to be ultimately reshaped by our 3D finite element enrichment plans in the design stages of remodelings with inter-adaptive refinements.

High-resolution proposal offers were brought off to a real parcelizations yielding well-posed object models at least total delay in total. Model shapes which were under certain surroundings are remodelled on the 2D contour grounds. Our specially-designed featured-refineries for the $3 \mathrm{D}$ rebuildings are cut into play by boundary condition adaptations. Boundary-adaptable refinements render the unprocessed models to higher dimensional states of our post-processings. Restorations of reformed models are restored in complete $3 \mathrm{D}$ regulations. All the processed models are tested in the finale of our 3-D restorative remakings. 


\section{References}

1. Hutchinson D, Hewitt T. Adaptive refinement for mass/spring simulations. Proceedings of the Eurographics Workshop on Computer Animation and Simulation; 1996. p. 31-45. https://doi.org/10.1007/978-3-7091-7486-9_3.

2. Taillefer F. Mixed modeling. Proceeding of Computer graphics; 1991. p. 467-78.

3. Numerical Recipes. 2019. https://en.wikipedia.org/wiki/ Numerical_Recipes.

4. Finte Element Procedures. 1996. http://web.mit.edu/kjb/ www/Books/FEP_2nd_Edition_4th_Printing.pdf.

5. Barbic J, James D. Real-time subspace integration for St. Venant-Kirchhoff deformable models. ACM Transactions on Graphics. 2005; 24(3):982-90. https://doi. org/10.1145/1073204.1073300.

6. Laplacian surface editing. 2004. https://igl.ethz.ch/projects/ Laplacian-mesh-processing/Laplacian-mesh-editing/laplacian-mesh-editing.pdf.

7. Rander W, Kanade T. Virtualized reality: Constructing timevarying virtual worlds from real world events. Proceedings.
Visualization; 1997. p. 277-84. https://doi.org/10.1109/ VISUAL.1997.663893.

8. Spectral geometry processing with Manifold Harmonics. 2008. http://www.cs.jhu.edu/ misha/ReadingSeminar/ Papers/Vallet08.pdf.

9. Introduction to implicit surfaces. 1997. https://www. amazon.com/Introduction-Implicit-Surfaces-KaufmannComputer/dp/155860233X.

10. Gleicher M, Witkin A. Differential manipulation. Graphics Interface; 1991. p. 61-7.

11. Polyhedra: Definition and Types. 1997. https://study.com/ academy/lesson/polyhedra-definition-types.html.

12. Shaping the future of fashion and apparel. 2019. http://www. optitex.com/.

13. Kim T, Vendrovsky E. Drivenshape: A data driven approach for shape deformation. ACM Computer Animation; 2008. p. 49-55. https://doi.org/10.1145/1401032.1401121.

14. Catmull E, Clark J. Recursively generated b-spline surfaces on arbitrary topological meshes. Computer-Aided Design; 1978.p. 350-5. https://doi.org/10.1016/00104485(78)90110-0. 